\title{
Immunogenicity of the BNT162b2 mRNA COVID-19 vaccine in patients with primary brain tumors: a prospective cohort study
}

\author{
Amir Massarweh $^{1} \cdot$ Roi Tschernichovsky $^{1}$ (1) $\cdot$ Amos Stemmer $^{2} \cdot$ Alexandra Benouaich-Amiel $^{3} \cdot$ Tali Siegal $^{3,4}$. \\ Noa Eliakim-Raz ${ }^{5,6} \cdot$ Salomon M. Stemmer ${ }^{1,7} \cdot$ Shlomit Yust-Katz ${ }^{3,7}$
}

Received: 19 October 2021 / Accepted: 22 November 2021 / Published online: 12 January 2022

(c) The Author(s), under exclusive licence to Springer Science+Business Media, LLC, part of Springer Nature 2021

\begin{abstract}
Purpose Immunogenicity of Covid-19 vaccines may be negatively impacted by anti-cancer treatment. The management of primary brain tumors (PBTs) routinely includes temozolomide and steroids, which are immune-suppressive. We aimed to determine the rate of seropositivity in PBT patients following receipt of two doses of the BNT162b2 vaccine.

Methods We prospectively evaluated IgG levels against SARS-CoV-2 spike protein in 17 PBT patients following two doses of the BNT162b2 vaccine. IgG levels were collected at two time points: T1—after a median of 44 days from the second vaccine dose and T2 - after a median of 130 days from the second dose. Titers were compared against a group of healthy controls (HC) comprised of patients' family members.

Results At T1, 88.2\% (15/17) of PBT patients achieved seroconversion, compared with 100\% (12/12) of HCs. Median IgG titer was significantly lower in the PBT group (1908 AU/mL vs 8,198 AU/mL; $\mathrm{p}=0.002)$. At T2, 80\% (12/15) of PBT patients seroconverted, compared to $100 \%(10 / 10)$ of HCs. Median IgG titer remained significantly lower in the PBT group (410 AU/mLvs $1687 \mathrm{AU} / \mathrm{mL} ; \mathrm{p}=0.002$ ). During the peri-vaccination period, 15 patients received systemic treatment and 8 patients were treated with corticosteroids. All 3 patients who failed to seroconvert at T2 were treated with corticosteroids. In a univariate analysis, steroid use was negatively associated with antibody titer.

Conclusion Most PBT patients successfully seroconvert following two doses of the BNT162b2 vaccine, albeit with lower antibody titer compared to HCs. Steroid use during the vaccination period is associated with lower titer.
\end{abstract}

Keywords Glioma $\cdot$ Covid-19 $\cdot$ Vaccine $\cdot$ mRNA $\cdot$ BNT162b2

Amir Massarweh and Roi Tschernichovsky have contributed equally to the study.

Roi Tschernichovsky

roits@ clalit.org.il

1 Department of Oncology, Davidoff Cancer Center, Rabin Medical Center, Beilinson Hospital, Petach Tikva, Israel

2 Department of Oncology, Sheba Medical Center, Tel Hashomer, Israel

3 Neuro-Oncology Unit, Davidoff Cancer Center, Rabin Medical Center, Beilinson Hospital, Petach Tikva, Israel

4 Faculty of Medicine, Hebrew University, Jerusalem, Israel

5 Department of Medicine E, Rabin Medical Center, Beilinson Hospital, Petach Tikva, Israel

6 Infectious Disease Unit, Rabin Medical Center, Beilinson Hospital, Petach Tikva, Israel

7 Sackler Faculty of Medicine, Tel Aviv University, Tel Aviv, Israel

\section{Introduction}

More than 18 months after the first reported cases of Covid19 , the global community is still struggling to curb the pandemic. Vaccines for the prevention of severe acute respiratory syndrome coronavirus 2 (SARS-CoV-2) infection are at the heart of this effort, with more than 4 billion doses administered globally as of August 2021 [1,2].

At the time of the initial vaccine rollouts, there was scarce data regarding efficacy in the oncology space. Patients with cancer were under-represented in the original phase III trials leading up to the approval of the BNT126b2 (Pfizer), mRNA-1273 (Moderna) and Ad26.COV2.S (Janssen) vaccines, and efficacy was not analyzed separately for this patient subgroup [3-5].

More recently, there have been reports indicating that the immunogenicity of the mRNA vaccine is attenuated in cancer patients, most notably in patients with hematological 
cancer actively receiving treatment [6-11]. In solid cancer patients, seroconversion rates appear to lag behind those of healthy controls over the two-dose schedule, and are accompanied by consistently lower IgG antibody titers [11, 12]. Importantly, titers are differentially impacted by different anti-neoplastic treatment modalities [10,11].

Given that immunogenicity seems to vary by anti-cancer treatment type, primary brain tumor patients and gliomas in particular are potentially at high risk for a sub-optimal response to vaccination. The treatment of glioma patients is multi-faceted and typically includes maximal surgical resection for operable patients, followed by post-operative radiation therapy. Temozolomide (TMZ) is administered in the adjuvant setting for low grade gliomas while concurrent and adjuvant temozolomide is routinely used in high grade tumors. TMZ, an alkylating agent, is known to cause hematological toxicity to a significant degree, including lymphopenia and neutropenia, which might hinder the humoral response to the vaccine [13-15]. Further contributing to immunosuppression in glioma patients is the frequent administration of glucocorticoids, commonly prescribed to reduce cerebral edema and alleviate the associated neurological symptoms [16]. In this regard, several studies have associated concomitant steroid usage with blunted immunogenicity to Covid-19 vaccines, and the Center for Disease Control (CDC) guidelines single out receipt of prednisone in a dose greater than $20 \mathrm{mg} /$ day for more than 14 days prior to vaccination as a putative risk factor for a reduced response [17-19].

So far, only a handful of primary brain tumor (PBT) cases were included in the pan-cancer studies evaluating immunogenicity of Covid-19 vaccines in cancer patients [7-10]. Furthermore, PBT patients' respective treatment modalities and their impact on seroconversion rates were not assessed. In this study, we aimed to shed further light on this pressing issue by using a brain tumor-only cohort of patients which have undergone a two-dose vaccination schedule with the BNT162b2 mRNA vaccine.

\section{Materials and methods}

\section{Study population}

The study population comprises a nested cohort of consecutive PBT patients, derived from a larger pan-cancer cohort recently reported on by our group [8].

The original cohort included adult patients $(>18$ years of age) with a histologically_confirmed diagnosis of solid malignancy, who were undergoing active anti-neoplastic treatment, had previously received 2 doses of the BNT162b2 mRNA Covid-19 vaccine, and were at least
30 days following the second ("boost") vaccination dose at the time of accrual.

Of 102 cancer patients in the original cohort, 9 patients were diagnosed with a PBT After summarizing the data from the original pan-cancer cohort, we increased the size of the PBT sub-cohort by enrolling 8 additional PBT patients through the neuro-oncology clinic at our institution. Treatments received by each patient were recorded at accrual (surgery, radiotherapy, temozolomide, bevacizumab, steroids, other).

The healthy control (HC) group was assembled from each cancer patient's respective family members/caregivers who accompanied him/her to the treatment center and had received 2 doses of BNT162b2. Exclusion criteria for both groups included history of prior Covid-19 infection (based on a positive polymerase chain reaction [PCR] test), active hematological cancer and pregnancy. Additional exclusion criteria for the control group included immune deficiency, active receipt of immunosuppressant therapy of any kind, and active other malignancy of any kind.

\section{Determination of serological status}

Blood samples were extracted from all participants at two separate time points. The first sample ("T1") was drawn at least 37 days after the second vaccine dose, between March 1 st 2021 and June 10th 2021. The second sample ("T2") was drawn at least three months after the first sample, between May 18th 2021 and July 22nd 2021.

Timepoint T1 was selected based on concurrent data showing that peak antibody levels are observed between days 7-30 following receipt of the second vaccination dose [20-22].

Serological status was determined using IgG antibody level against the SARS-CoV-2 spike receptor-binding domain, quantified using a chemiluminescent microparticle immunoassay (by Abbott@). An antibody level of $50 \mathrm{AU} /$ $\mathrm{mL}$ or higher was considered positive for successful seroconversion [23].

\section{Statistical analysis}

Univariate and multivariable analyses were performed by fitting a generalized linear model on the $\log$ of $\operatorname{IgG}$ values at $\mathrm{T} 2$ and included age and days after vaccination as continuous variables, and sex, treatments, and steroid use at vaccination as categorical variables. The Spearman correlation method was used to assess the correlation between the $\mathrm{IgG}$ values and the number of days after vaccination. The difference in IgG values between patients and controls was evaluated using the Wilcoxon rank sum test. A $P$ value $<0.05$ was 
considered significant. Statistical analysis was performed using R, version 4.0.2 (R Foundation).

\section{Ethics statement}

The study was approved by the Rabin Medical Center Ethics Committee and all patients as well as HC provided informed consent.

\section{Results}

\section{Characteristics of study population}

A total of 17 PBT patients and $12 \mathrm{HCs}$ were included in the study (see Table 1). The PBT group comprised 11 male

Table 1 Study population

\begin{tabular}{lll}
\hline & PBT $(\mathrm{n}=17)$ & $\mathrm{HC}(\mathrm{n}=12)$ \\
\hline $\begin{array}{l}\text { Age (median [IQR]) } \\
\text { Sex n (\%) }\end{array}$ & $65(58-71)$ & $58(48-69)$ \\
Male & $11(65 \%)$ & $2(17 \%)$ \\
Female & $6(35 \%)$ & $10(83 \%)$ \\
Diagnosis n (\%) & $13(76 \%)$ & $\mathrm{n} / \mathrm{a}$ \\
Glioblastoma & $2(12 \%)$ & $\mathrm{n} / \mathrm{a}$ \\
Anaplastic astrocytoma & $1(6 \%)$ & $\mathrm{n} / \mathrm{a}$ \\
Oligodendroglioma & $1(6 \%)$ & $\mathrm{n} / \mathrm{a}$ \\
Atypical meningioma & $5(27 \%)$ & $\mathrm{n} / \mathrm{a}$ \\
Treatment components in the three months prior to vaccination \\
Radiation & $11(65 \%)$ & $\mathrm{n} / \mathrm{a}$ \\
Temozolomide & $4(23 \%)$ & $\mathrm{n} / \mathrm{a}$ \\
Bevacizumab & $1(6 \%)$ & $\mathrm{n} / \mathrm{a}$ \\
Clinical trial drug & $2(12 \%)$ & $\mathrm{n} / \mathrm{a}$ \\
Surgery & $8 / 17(47 \%)$ & $\mathrm{n} / \mathrm{a}$ \\
Corticosteroids at time of vaccination & $\mathrm{n} / \mathrm{a}$ \\
Corticosteroid (Dexamethasone) dos- & $\mathbf{0 . 5 ( 0 - 1 6 )}$ \\
age (mean mg [range]) & & \\
\hline
\end{tabular}

$I Q R$ interquartile range patients, with a median age of 65 (58-71). In the HC group, 2 participants were male and the median age was 58 (48-69). Most patients in the PBT group had a glioma diagnosis (glioblastoma multiforme $=13$, anaplastic astrocytoma $=2$, oligodendroglioma $=1$ ). A single patient with a non-glioma diagnosis (atypical meningioma) was included based on having been treated with bevacizumab, a drug commonly prescribed for glioma in the second-line setting.

\section{Serological outcome}

At timepoint T1, the median elapsed time in days from the 2nd vaccination dose was 44 (37-53) for the PBT group and 44 (34-51) for the HC group $(\mathrm{p}=0.8)$ (see Table 2). At this timepoint, $88.2 \%$ (15/17) of PBT patients achieved seroconversion, compared with $100 \%$ (12/12) of HCs. Median IgG titer levels were significantly lower in the PBT group compared to the HC group (1908 AU/mL vs $8198 \mathrm{AU} / \mathrm{mL}$, respectively; $\mathrm{p}=0.002$ ) (see Table 2; Fig. 1 ).

At $\mathrm{T} 2$, the median elapsed time in days from 2 nd vaccination was 130 (126-138) and 134 (126-140) for the PBT and $\mathrm{HC}$ groups, respectively. IgG levels were available for 10 out of 12 patients in the HC group, with a seroconversion rate of $100 \%$ (10/10). In the PBT group, IgG level were available for 15 out of 17 patients, with a seroconversion rate of $80 \%(12 / 15)$. Two PBT patients who were seronegative at $\mathrm{T} 1$ remained so at T2. One PBT patient who successfully seroconverted at $\mathrm{T} 1$ (IgG level of $189 \mathrm{AU} / \mathrm{mL}$ ) reverted back to a negative status at T2 (IgG level of $42 \mathrm{AU} / \mathrm{mL}$ ). Median IgG titers remained significantly lower in the PBT group (410 vs 1687; $p=0.002$ ) (see Fig. 1).

Analysis of $\operatorname{IgG}$ levels as a function of time elapsed from 2nd vaccination at $\mathrm{T} 2$ revealed significant inverse correlations for both groups (see Fig. 2).

\section{Effect of concomitant treatments during the vaccination period}

Eleven patients received TMZ and four patients received bevacizumab over the course of the vaccination schedule.

Table 2 Serological status

\begin{tabular}{llll}
\hline & PBT & HC & p-value \\
\hline Timepoint T1 & & & \\
$\quad$ Days post-vaccination (median [IQR]) & $44(37-53)$ & $44(34-51)$ & 0.8 \\
Seroconversion Rate (\%) & $88.2 \%(15 / 17)$ & $100 \%(12 / 12)$ & \\
IgG Titer Values (AU/mL) (median [IQR]) & $1908(471-4,387)$ & $8198(4,515-12,377)$ & 0.002 \\
Timepoint T2 & $130(126-138)$ & $134(126-140)$ & $>0.9$ \\
Days post-vaccination (median [IQR]) & $80 \%(12 / 15)$ & $100 \%(12 / 12)$ & \\
Seroconversion Rate $(\%)$ & $410(138-770)$ & $1687(937-2,326)$ & 0.002 \\
IgG Titer Values (AU/mL) (median [IQR])
\end{tabular}

$I Q R$ interquartile range 

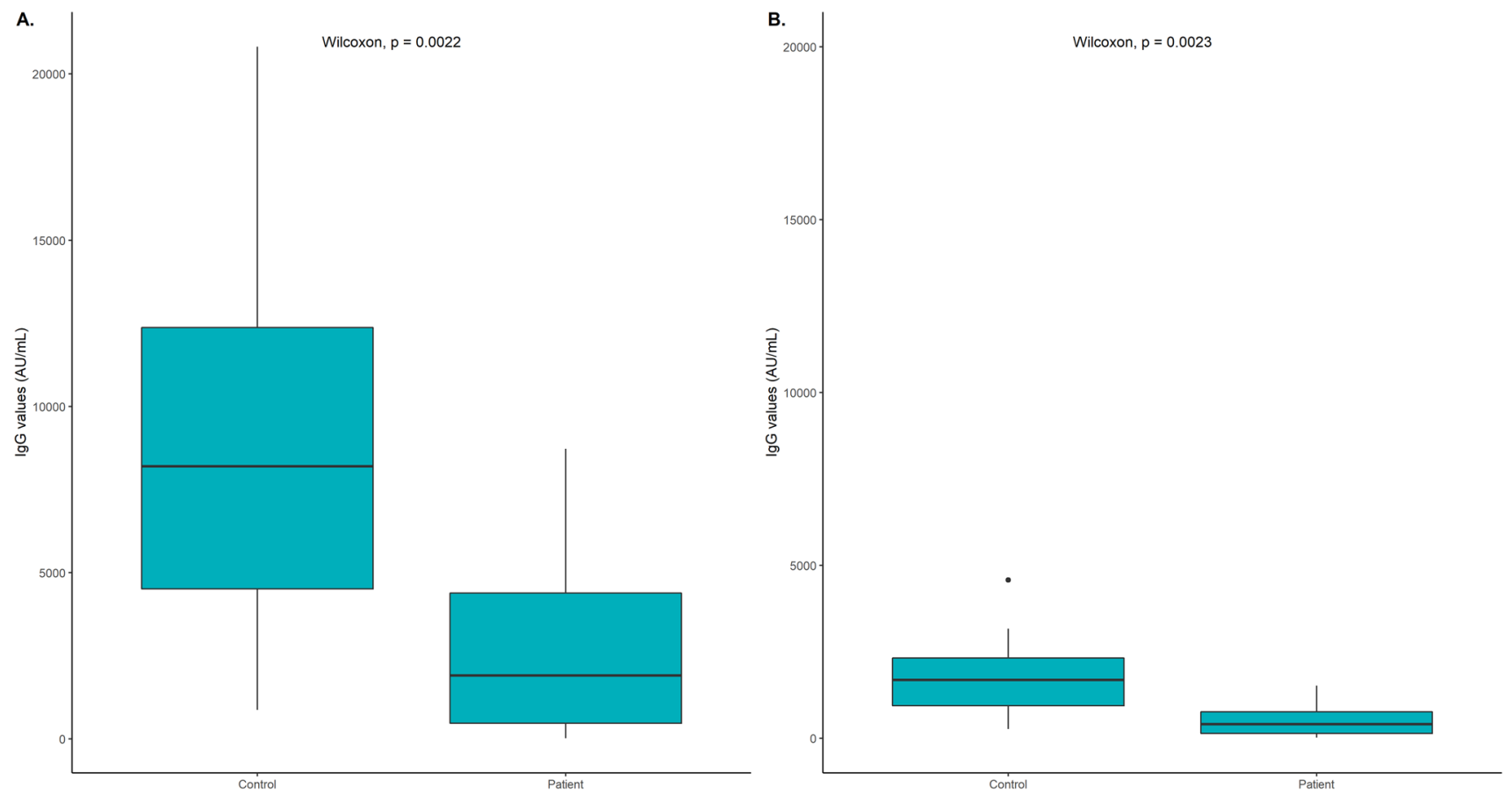

Fig. 1 IgG titer levels
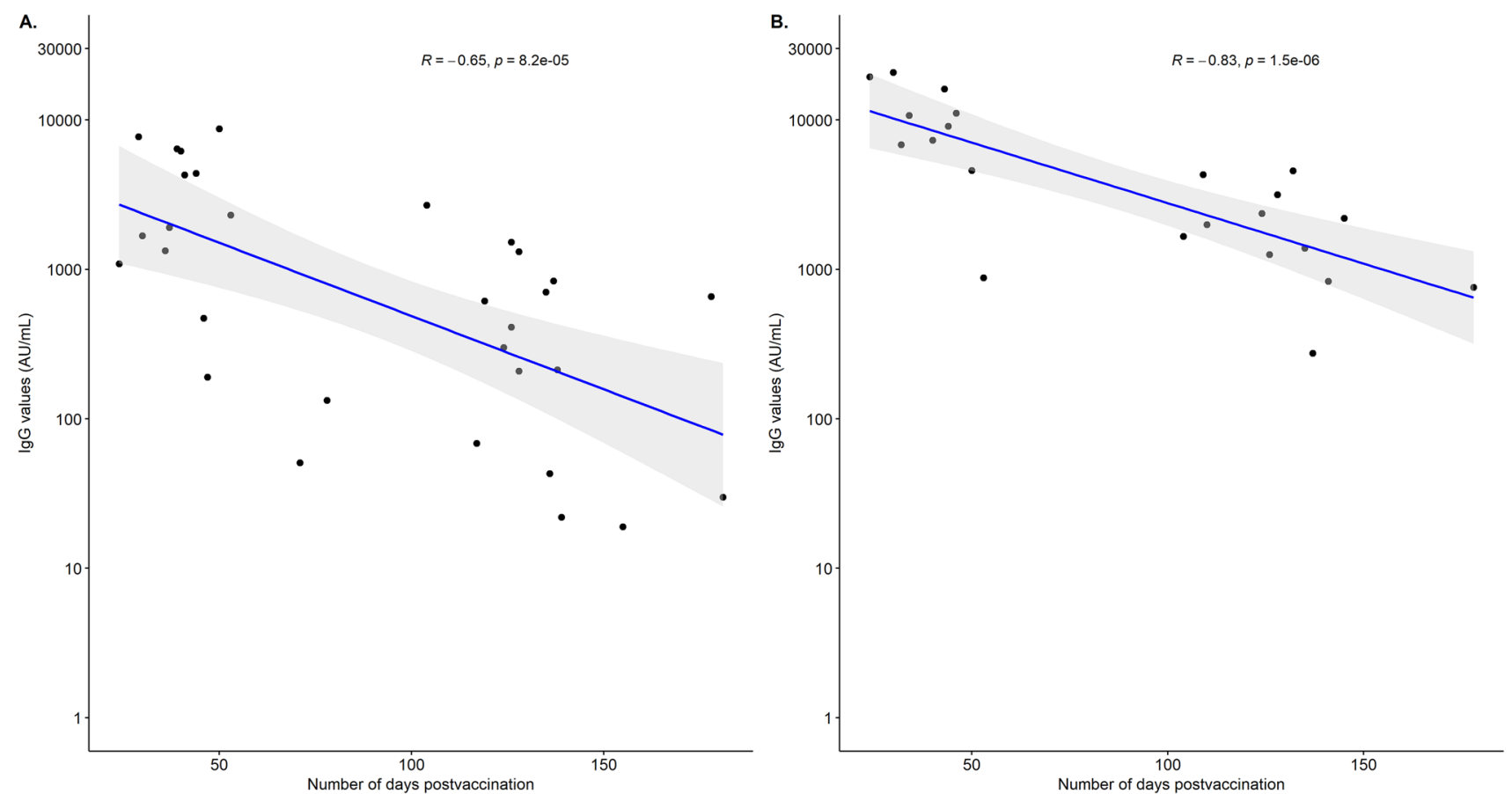

Fig. 2 IgG titer levels versus time elapsed in days from second vaccination dose

Eight patients were under systemic glucocorticoid treatment at the time of vaccination.

Notably, all three PBT patients who failed to achieve seroconversion at $\mathrm{T} 2$ had been treated with corticosteroids, with two patients out of the three receiving high-dose dexamethasone (10 mg and $16 \mathrm{mg}$, respectively). Additionally, all three patients were under active anti-neoplastic treatment; two patients had received adjuvant TMZ treatment, and one patient received bevacizumab. 
Of the PBT patients who successfully seroconverted at $\mathrm{T} 2$, four patients were under steroid treatment, however all four were treated with low doses (between 0.5 and $4 \mathrm{mg}$ ).

In a univariate analysis at timepoint $\mathrm{T} 2$, corticosteroid use was negatively associated with diminished $\operatorname{IgG}$ titers. The association was not significant in a multivariable analysis. Male sex was positively associated with IgG titers. No additional putative risk factors for diminished titers were identified, including treatment with TMZ or bevacizumab (see Table 3).

\section{Adverse events}

Over a median follow-up period of 6 months, no adverse events were observed in either group following vaccination, including hematological toxicity or venous thromboembolism.

\section{Discussion}

To our knowledge, this is the largest-reported cohort of PBT patients assessed for serological status following two doses of the BNT162b2 mRNA Covid-19 vaccine. Eighty percent of PBT patients in our study seroconverted followings a twodose vaccination schedule, which is a lower rate than was previously recorded in pan-cancer cohorts, including from our group [11, 12]. As with the previous pan-cancer cohorts, median IgG titers in the PBT group were significantly lower compared with $\mathrm{HCs}$, and remained lower upwards of 4 months post-vaccination.

The reduced immunogenicity observed in the PBT cohort should be interpreted with caution. First, while there is an agreed-upon laboratory $\operatorname{IgG}$ value above which a person is considered to have successfully seroconverted, the threshold required for actual real-life clinical protection against infection (a "protective threshold") has not been established [24]. In addition, whether or not lower IgG titers in seroconverted individuals necessarily confer greater risk of infection is also unclear. Early data does indicate, however, that breakthrough infections are correlated with lower peak levels of both neutralizing and S- specific IgG antibodies. This was recently demonstrated in a study from Israel conducted among vaccinated healthcare personnel [25].

All three PBT patients who failed to successfully seroconvert at $\mathrm{T} 2$ were under daily corticosteroid treatment during the vaccination schedule, with two patients receiving high-dose dexamethasone. Indeed, a negative association between steroid treatment and $\mathrm{IgG}$ titer was confirmed in a univariate analysis. This finding mirrors previously reported data linking concomitant steroid usage with impaired immunogenicity to Covid-19 vaccines in other, non-cancer patient populations [17, 18, 26-28]. For example, Rabinowich et al. evaluated a cohort of 80 liver transplant (LT) patients for anti-spike protein IgG levels 10-20 days following the second dose of BNT162b2, and found a significantly reduced seroconversion rate compared to $\mathrm{HCs}(47.5 \%)$, for which

Table 3 Univariate and multivariable analysis at timepoint T2

\begin{tabular}{|c|c|c|c|c|c|c|}
\hline \multirow[b]{2}{*}{ Characteristic } & \multicolumn{3}{|c|}{ Univariate analysis } & \multicolumn{3}{|c|}{ Multivariate analysis } \\
\hline & Beta & $95 \% \mathrm{CI}$ & p-value & Beta & $95 \% \mathrm{CI}$ & p-value \\
\hline Age & -0.03 & $-0.08,0.03$ & 0.3 & 0.01 & $-0.03,0.05$ & 0.6 \\
\hline \multicolumn{7}{|l|}{ Sex } \\
\hline Female & - & - & & - & - & \\
\hline Male & 1.5 & $0.22,2.8$ & 0.039 & 1.7 & $0.91,2.5$ & 0.014 \\
\hline \multicolumn{7}{|l|}{ Treatments } \\
\hline Bevacizumab & - & - & & - & - & \\
\hline Surgery & 1.6 & $-0.92,4.1$ & 0.2 & 1.0 & $-1.2,3.2$ & 0.4 \\
\hline Temozolomide & -0.36 & $-2.2,1.4$ & 0.7 & -0.2 & $-0.93,0.54$ & 0.6 \\
\hline Elapsed time from vaccination (days) & -0.03 & $-0.06,0.01$ & 0.2 & -0.01 & $-0.02,0.01$ & 0.5 \\
\hline \multicolumn{7}{|l|}{ Steroid treatment at vaccination } \\
\hline No & - & - & & - & - & \\
\hline Yes & -1.9 & $-3.0,-0.82$ & 0.005 & -1.1 & $-1.9,-0.25$ & 0.063 \\
\hline \multicolumn{7}{|l|}{ Diagnosis } \\
\hline Anaplastic Astrocytoma & - & - & & - & - & \\
\hline Atypical meningioma & 0.22 & $-3.2,3.7$ & $>0.9$ & 2.4 & $0.51,4.2$ & 0.066 \\
\hline Glioblastoma multiforme & -1.3 & $-3.4,0.91$ & 0.3 & 0.37 & $-1.1,1.9$ & 0.7 \\
\hline Oligodendroglioma & 0.67 & $-2.8,4.1$ & 0.7 & 0.08 & $-1.4,1.5$ & $>0.9$ \\
\hline
\end{tabular}


high-dose steroids given in the previous 12 months was a significant predictor [26]. Similarly, in a multi-center study of 478 patients with auto-immune systemic diseases, several patient sub-groups had an increased prevalence of vaccine non-responsiveness, including the sub-group of patients treated with glucocorticoids [27]. In the study, steroid usage was predictive of non-responsiveness in a univariate analysis (OR:2.27), but the effect failed to replicate in the subsequent multivariate analysis. Notably, while four PBT patients in our study successfully seroconverted whilst on active steroid treatment, none required dosing above $4 \mathrm{mg}$, suggesting the level of impairment may be dose-dependent. In the abovementioned LT patient cohort, low-dose steroid usage trended towards impaired vaccine response, but not to a statistically significant degree.

In contrast to our findings regarding steroids, there was no demonstrable association between receipt of TMZ in the peri-vaccination period and subsequent IgG titer levels. TMZ is known to induce lymphopenia which is driven primarily by selective CD4 + T-Cell Depletion [15, 29]. Therefore, any impairment to vaccine response exerted by TMZ would presumably be mediated through reduced CD4 + counts. In our study, longitudinal CD4 counts across the vaccination schedule were not routinely obtained, precluding a correlation analysis to be performed between $\mathrm{CD} 4+$ levels and humoral response. A relevant extrapolation could be made from the limited published data on vaccinated persons with Human Immunodeficiency Virus (HIV). Frater and colleagues recently reported safety and efficacy outcomes for a subset of $54 \mathrm{HIV}$-positive patients included in the phase II/III study of the ChAdOx1 $\mathrm{nCoV}-19$ vaccine (Astra-Zeneca) [30]. In the study, all participants were on active anti-retroviral therapy, had undetectable HIV viral load, and the median CD4 counts at enrollment were 694 cells $/ \mu \mathrm{L}$. The authors found no difference in the magnitude or durability of anti-spike IgG response between HIV-positive and HIV-negative participants, and there was no correlation between CD4 counts and the level of $\operatorname{IgG}$ response at day 56 following the prime vaccination (first dose).

Our study suffers from several limitations. Primarily, small sample sizes in both groups prohibit making robust conclusions regarding vaccine immunogenicity in PBT patients. Secondly, the groups were not matched by age and sex - the proportion of males in the PBT group was higher, as was the median age. Lastly, cellular immunity measureswhich are of relevance in patients receiving lymphocytemodulating treatment, were not assessed.

Notwithstanding the above limitations, our findings do point to a reduced vaccine response in PBT patients compared with healthy controls, as evidenced by a lower rate of successful seroconversion, as well as reduced IgG levels over a prolonged follow-up period. Both of these effects might be mediated by chronic steroid use throughout the vaccination schedule. In light of our findings, it appears prudent to minimize corticosteroid treatment for PBT patients during the peri-vaccination period to the lowest-possible dose required to control edema-related symptoms. In addition, PBT patients should be strongly considered for receipt of a third ("booster") vaccination dose. Alternatively, IgG titer levels may be serially monitored following the two-dose schedule to single out the subset of PBTs patients who serve to benefit from a booster dose.

Like many other facets of oncology care, the treatment of neuro-oncology patients has been severely disrupted by the pandemic [31]. Minimizing infection risk should be a high priority especially in patients with high — grade glioma, who typically harbor multiple risk factors for severe infection including older age, continuous immunosuppression, and reduced mobility secondary to neurological damage.

Author contributions Substantial contribution to conception and design: NER, SMS, SYK. Acquisition of data: AM, ABA, SMS, SYK. Analysis and interpretation of data: AM, RT, AS, NER, SMM, SYK. Drafting of article: RT, AS, NER, SMS, SYK. Critical revision of article: RT, SMS, SYK. Final approval of version to be published: NER, SMS, SYK, RT.

Funding No funding source to declare.

Data availability The datasets generated during and/or analysed during the current study are available from the corresponding author on reasonable request.

\section{Declarations}

Conflict of interest The authors declare that they have no financial or non-financial interests that are directly or indirectly related to the work submitted for publication.

\section{References}

1. Holder J. Tracking coronavirus vaccinations around the world. The New York Times. https://www.nytimes.com/interactive/ 2021/world/covid-vaccinations-tracker.html. Published January 29, 2021. Accessed 6 Aug 2021.

2. Lopez Bernal J, Andrews N, Gower C, et al. Effectiveness of Covid-19 vaccines against the B.1.617.2 (Delta) Variant. N Engl J Med. 2021. https://doi.org/10.1056/NEJMoa2108891

3. Polack FP, Thomas SJ, Kitchin N et al (2020) Safety and efficacy of the BNT162b2 mRNA Covid-19 vaccine. N Engl J Med 383(27):2603-2615. https://doi.org/10.1056/NEJMoa2034577

4. Baden LR, El Sahly HM, Essink B et al (2021) Efficacy and safety of the mRNA-1273 SARS-CoV-2 vaccine. N Engl J Med 384(5):403-416. https://doi.org/10.1056/NEJMoa2035389

5. Sadoff J, Gray G, Vandebosch A et al (2021) Safety and efficacy of single-dose Ad26.COV2.S vaccine against Covid-19. N Engl J Med 384(23):2187-2201. https://doi.org/10.1056/NEJMoa2101 544 
6. Herishanu Y, Avivi I, Aharon A et al (2021) Efficacy of the BNT162b2 mRNA COVID-19 vaccine in patients with chronic lymphocytic leukemia. Blood J Am Soc Hematol 137(23):31653173. https://doi.org/10.1182/blood.2021011568

7. Monin L, Laing AG, Muñoz-Ruiz M et al (2021) Safety and immunogenicity of one versus two doses of the COVID-19 vaccine BNT162b2 for patients with cancer: interim analysis of a prospective observational study. Lancet Oncol 22(6):765-778. https://doi.org/10.1016/S1470-2045(21)00213-8

8. Massarweh A, Eliakim-Raz N, Stemmer A et al (2021) Evaluation of seropositivity following BNT162b2 messenger RNA vaccination for SARS-CoV-2 in patients undergoing treatment for cancer. JAMA Oncol. https://doi.org/10.1001/jamaoncol.2021.2155

9. Palich R, Veyri M, Vozy A et al (2021) High seroconversion rate but low antibody titers after two injections of BNT162b2 (PfizerBioNTech) vaccine in patients treated with chemotherapy for solid cancers. Ann Oncol. https://doi.org/10.1016/j.annonc.2021.06.018

10. Addeo A, Shah PK, Bordry N et al (2021) Immunogenicity of SARS-CoV-2 messenger RNA vaccines in patients with cancer. Cancer Cell. https://doi.org/10.1016/j.ccell.2021.06.009

11. Eliakim-Raz N, Massarweh A, Stemmer A, Stemmer SM (2021) Durability of response to SARS-CoV-2 BNT162b2 vaccination in patients on active anticancer treatment. JAMA Oncol. https://doi. org/10.1001/jamaoncol.2021.4390

12. Goshen-Lago T, Waldhorn I, Holland R et al (2021) Serologic status and toxic effects of the SARS-CoV-2 BNT162b2 vaccine in patients undergoing treatment for cancer. JAMA Oncol. https:// doi.org/10.1001/jamaoncol.2021.2675

13. Kourelis TV, Buckner JC, Gangat N, Patnaik MM (2015) Temozolomide induced bone marrow suppression-a single institution outcome analysis and review of the literature. Am J Hematol 90(9):E183-E184. https://doi.org/10.1002/ajh.24066

14. Gerber DE, Grossman SA, Zeltzman M, Parisi MA, Kleinberg L (2007) The impact of thrombocytopenia from temozolomide and radiation in newly diagnosed adults with high-grade gliomas1. Neuro Oncol 9(1):47-52. https://doi.org/10.1215/15228 517-2006-024

15. Grossman SA, Ye X, Lesser G et al (2011) Immunosuppression in patients with high-grade gliomas treated with radiation and temozolomide. Clin Cancer Res 17(16):5473-5480. https://doi. org/10.1158/1078-0432.CCR-11-0774

16. Wong ET, Swanson KD (2019) Dexamethasone-friend or foe for patients with glioblastoma? JAMA Neurol 76(3):247. https://doi. org/10.1001/jamaneurol.2018.4530

17. Ruddy JA, Connolly CM, Boyarsky BJ et al (2021) High antibody response to two-dose SARS-CoV-2 messenger RNA vaccination in patients with rheumatic and musculoskeletal diseases. Ann Rheum Dis 80(10):1351-1352. https://doi.org/10.1136/annrh eumdis-2021-220656

18. Bugatti S, De Stefano L, Balduzzi S et al (2021) Methotrexate and glucocorticoids, but not anticytokine therapy, impair the immunogenicity of a single dose of the BNT162b2 mRNA COVID19 vaccine in patients with chronic inflammatory arthritis. Ann Rheum Dis. https://doi.org/10.1136/annrheumdis-2021-220862

19. Centers for Disease Control and Prevention. Updated healthcare infection prevention and control recommendations in response to COVID-19 vaccination. https://www.cdc.gov/coronavirus/ 2019-ncov/hcp/infection-control-after-vaccination.html. Accessed 8 Aug 2021

20. Falsey AR, Frenck RW, Walsh EE et al (2021) SARS-CoV-2 neutralization with BNT162b2 vaccine dose 3. N Engl J Med 385(17):1627-1629. https://doi.org/10.1056/NEJMc2113468

21. Levin EG, Lustig $Y$, Cohen $C$ et al (2021) Waning immune humoral response to BNT162b2 Covid-19 vaccine over 6 months. N Engl J Med. https://doi.org/10.1056/nejmoa2114583

22. Sahin U, Muik A, Vogler I et al (2020) BNT162b2 induces SARSCoV-2-neutralising antibodies and $\mathrm{T}$ cells in humans. MedRixv. https://doi.org/10.1101/2020.12.09.20245175

23. Meschi S, Colavita F, Bordi L et al (2020) Performance evaluation of Abbott ARCHITECT SARS-CoV-2 IgG immunoassay in comparison with indirect immunofluorescence and virus microneutralization test. J Clin Virol 129:104539. https://doi.org/10. 1016/j.jcv.2020.104539

24. Krammer F (2021) Correlates of protection from SARS-CoV-2 infection. The Lancet 397(10283):1421-1423. https://doi.org/10. 1016/S0140-6736(21)00782-0

25. Bergwerk M, Gonen T, Lustig Y et al (2021) Covid-19 breakthrough infections in vaccinated health care workers. $\mathrm{N}$ Engl $\mathrm{J}$ Med. https://doi.org/10.1056/NEJMoa2109072

26. Rabinowich L, Grupper A, Baruch R et al (2021) Low immunogenicity to SARS-CoV-2 vaccination among liver transplant recipients. J Hepatol 75(2):435-438. https://doi.org/10.1016/j. jhep.2021.04.020

27. Ferri C, Ursini F, Gragnani L et al (2021) Impaired immunogenicity to COVID-19 vaccines in autoimmune systemic diseases high prevalence of non-response in different patients' subgroups. J Autoimmun 125:102744. https://doi.org/10.1016/j.jaut.2021. 102744

28. Furer V, Eviatar T, Zisman D et al (2021) Immunogenicity and safety of the BNT162b2 mRNA COVID-19 vaccine in adult patients with autoimmune inflammatory rheumatic diseases and in the general population: a multicentre study. Ann Rheum Dis 80(10):1330-1338. https://doi.org/10.1136/annrh eumdis-2021-220647

29. Huang J, DeWees TA, Badiyan SN et al (2015) Clinical and dosimetric predictors of acute severe lymphopenia during radiation therapy and concurrent temozolomide for high-grade glioma. Int J Radiat Oncol Biol Phys 92(5):1000-1007. https://doi.org/10. 1016/j.ijrobp.2015.04.005

30. Frater J, Ewer KJ, Ogbe A et al (2021) Safety and immunogenicity of the ChAdOx1 nCoV-19 (AZD1222) vaccine against SARSCoV-2 in HIV infection: a single-arm substudy of a phase $2 / 3$ clinical trial. Lancet HIV 8(8):e474-e485. https://doi.org/10.1016/ S2352-3018(21)00103-X

31. Mohile NA, Blakeley JO, Gatson NTN et al (2020) Urgent considerations for the neuro-oncologic treatment of patients with gliomas during the COVID-19 pandemic. Neuro Oncol 22(7):912917. https://doi.org/10.1093/neuonc/noaa090

Publisher's Note Springer Nature remains neutral with regard to jurisdictional claims in published maps and institutional affiliations. 